\title{
Development of Passengers Vehicle Overloading Control System
}

\author{
Phesto Peter Namayala $^{1}$ Sospeter Iganja Gabriel $^{2}$ Godfrey kayombo $^{3} \&$ Peter \\ Kambosha $^{4}$ \\ Department of Information and Communication Technology $1,3,4$ \\ Department of Electronics and Telecommunication Engineering ${ }^{2}$ \\ Mbeya University of Science and Technology \\ Box 131, Mbeya, Tanzania.
}

\begin{abstract}
Passengers overloading control has been a challenge in Tanzania, with the current practices checkup for overloading is done at special inspections points, leaving loopholes to both drivers and conductor deploy alternative ways to circumvent burdens. Sophisticated measures are required in controlling public buses' overloading because they can help in reducing deaths and casualties as the findings show that excessive passengers is one of the major causes of many deaths and casualties when accidents occur. There are a number of existing technics for passengers' overloading control in public busses, counting of passengers, video processing using the zenithal camera for capturing the bio-directional passengers flow, infra-red motion analyzer and stereovision are commonly used. The current practice of checking and controlling passengers' overloading which is done by stationed police officers and SUMATRA is associated with many challenges and limitations, being labor-intensive, not providing real-time data, being reactive rather than proactive, not applicable in rainy and night time, encourage bribery and lacking control are some of the commonly identified problems and limitations.
\end{abstract}

This research concentrates with the design and develops a sophisticated embedded passengers'vehicles overloading system that monitors and automatically controlling the overloading process. Development was possible through the use of ADCUM Camera, Diode 1N4007, Arduino UNO R3, GSM modem A6, SIM900 Liquid crystal display 16X4, DC motor, Relay 5v, Regulator 7805 and battery $4 A H$.

Both qualitative and quantitative design approach was used when undertaking this research, a stratified sampling technique was used when collecting data and to present findings SPSS was used to analyze data.

Key Words: Tanzania, passenger's vehicles, overloading, control, MUST.

\subsection{INTRODUCTION}

\subsection{Situation analysis}

Passengers overloading control has been a challenge in Tanzania, with the current practices checkup for overloading is done at special inspections points, leaving loopholes to both drivers and conductors deploy alternative ways to circumvent burdens. It has been a common practice for drivers and conductors to offload passengers and propel them ahead using alternative means of transport when they are about to reach an inspection point. The current inspection system doesn't work efficiently as the check is done at random, and it is difficult during rainy seasons and at night times, it is also costly and needs immense labor power [1]. Sophisticated measures are required in controlling public buses' overloading because they can helps in reducing deaths and casualties as the findings show that excessive passengers is one of the major causes of many deaths and casualties when accidents occur. Passengers when travelling are required to sit and wear seat belts for their safety [2]. A single seat belt cannot be tighten to multiple passengers, current overloading lets passengers seat than recommended seating capacity and some are even travelling without a seat. Overloading passenger in public transport is a problem in many regions within the country. Practice has fully proved that the efforts that came only from policy to control overloading of passenger vehicles is not enough, which made the work of relevant departments seem rather passive [3]. Passenger overloading carries costs in terms of lives, property and maintenance of buses [1]. Overloading passengers in buses is illegal which carries with range of risks and penalties [3]. There are a number of existing technics for passengers' overloading control in public busses, counting of passengers, video processing using zenithal camera for capturing the bio-directional passengers flow, infra-red motion analyzer and stereovision are commonly used. 
Passenger counting can be very useful for the management, scheduling and planning of the public transport, since it enables to set the transport service according to the observed demand, in order to improve the level of service provided to the users [4]. Though internationally various passengers counting technologies are on market today, Tanzania as a country has been passive to adopt passengers counting systems.

In this research we argue that the use of sophisticated Passenger's Vehicle Overloading Control is linked with a number of paybacks, adequate planning, provisions of accurate data, real time monitoring, bribery preventions and likelihood of instituting control measures comprising switching off the engine and prevent it from start when a particular vehicle is overloaded. High season is the appropriate season endorsed for using Passenger's Vehicle Overloading Control System. Many researchers are working on different researches to overcome challenges of public transport overloading, Shanzhen XU and Qian ZHAO (2011) researched and designed AT89C51 microcontroller based passenger overloading control system, the system was vehicle-mounted and very convenient to install.

\subsection{Problem Statement}

In Tanzania passenger overloading has persevered being a serious problem in public transport, example a bus capable of carrying fifteen (15) passengers may be loaded up to thirty (30) passengers or more. The current practice of checking and controlling passengers' overloading which is done by stationed police officers and SUMATRA is associated with many challenges and limitations, being labor intensive, not providing real time data, being reactive rather than proactive, not applicable in rainy and night time, encourage bribery and lacking control are some of the commonly identified problems and limitations.

The outcome of this research was to design and develop a sophisticated embed passengers' vehicles overloading system that monitor and automatically controlling the overloading process,; both drivers and conductor are alerted about the number of bordered passengers and when the alert is ignored the system sends both vehicle's location and contained number of passengers to both control authorities and vehicle's owner. As the measure of ensuring every passenger is able to wear seat belts the system also prevent vehicle from starting when bordered passenger exceeds the seating capacity.

\subsection{LITERATURE REVIEW}

The considerable development of passengers' traffic in public transportation has made it indispensable to set up specific methods of organization and management for a proper loading [5]. Most of the bus operators companies are very much concerned with counting passengers, which allows improved diagnosis of fraud, optimization of line management, traffic control and forecast, budgetary distribution between the different lines, and improvements in the quality of service [6]. In South Africa a number of automatic passengers counting systems have been used and they overall provide accuracy of ninety nine percent (99\%), according to the key technology used for counting, systems are grouped as Infrared (IR) beams, Infrared optic sensors, Low ultrasonic frequency sensors, Treadle mats. (Load cells) and Video camera.

In this research designing of a system is concerning with capturing input signal by camera that used to catch images of passenger within the bus after that images are analyzed for determining whether the number of bordered passengers found are in compliance with the seating capacity. After analysis the system is then able to make decisions, made decisions are sending messages to both bus owner and overloading control authorities, display total number of passengers for drivers and passengers to see and in extreme case turn off fuel injection and prevent the bus from starting.

Apart from solving the overloading problem, the research also overcome problem of other existing systems counting passengers when getting in/out at the bus's door, Counting passengers at the bus's door ignore those using illegal doors, example the system is able to take care of passengers who have used windows or driver's door when getting in/out of the bus. Many researchers have reported image-processing based methods for counting people passing through a door or gate to have occlusion, overlapping, merge-split, and shadow effects [7]. To accurately count passengers getting in and out of a bus, the major difficulty is how to cope with both problems of camera shaking and variation of illumination that will seriously affect the frame-difference techniques for moving-object segmentation [8].

\subsection{Research Significance}

The significances of this research are to assess the existing situation on the problem of the vehicles' overloading in public transport in Tanzania, design and develop vehicles' overloading system that addresses many of the existing challenges and 
limitations. The designed and developed system is able to reduce cost and manpower, works 24/7 and for any type of weather, provide real time data, stops the bribe value chain, proactive and provide linkage with both owners and control authorities.

\subsection{RESEARCH OBJECTIVES}

Objective under this research are categorized into two categories which are main objective and specific objectives.

\subsection{Main Objective}

To develop a Passengers` Vehicle Overloading Control System

\subsection{Specific objectives}

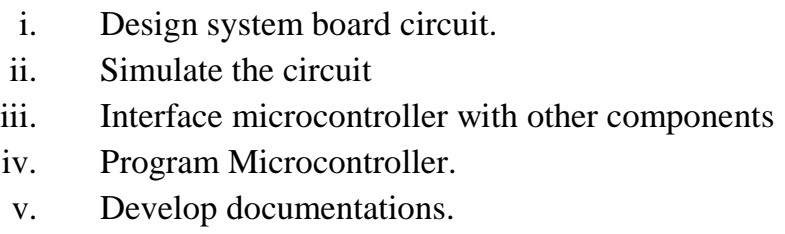

\subsection{Scope and Limitation of Project}

The scope of this research is to design, simulate and develop a vehicles' overloading control system. Developed system uses video processing technique captured using zenithal camera and only work if there is necessary lighting requirements. The system is limited by areas where cameras are installed. The system is also limited by the presence of GSM networks to operative throughput.

\subsection{METHODOLOGY}

From researchers methodology includes the design, setting, sample, methodological limitations, and the data collection and analysis techniques in a study [9]. In the research design both qualitative and quantitate approaches were used so as to supplement weaknesses of each other, stratified random sampling was used to establish sample from the population. There are many ways used to draw samples from a population, we intuitively think of a good sample as one that is representative of the population from which the sample has been drawn. By 'representative' we do not necessarily mean the sample matches the population in terms of observable characteristics, but rather that the results from the data we collect from the sample are consistent with the results we would have obtained if we had collected data on the entire population [10]. To collect data structured questionnaire were used as the data collection tool, data collection is important in research as it allows the delivery of accurate information requirements and development of meaningful projects. The methods that are normally used to collect the data are questionnaire, internet surfing, reading different books and consultation of some experts [11]. SPSS software was used to analyze gathered data and present findings.

\subsection{System design}

For ensuring proper systems design and communicate both system' structure, behavior, and interaction with external entities to other stakeholder a combination of modern approach of OOP design with UML and traditional approach were chosen. The design

\subsubsection{Block diagram}

A block diagram is a diagram showing schematic form of the general arrangement of the parts or components of a complex system or process, such as an industrial apparatus or an electronic circuit. It is usually used to show general arrangement of a system and main components used in formulating the system. Figure 1 below shows the block diagram of the developed passenger's overloading control system 


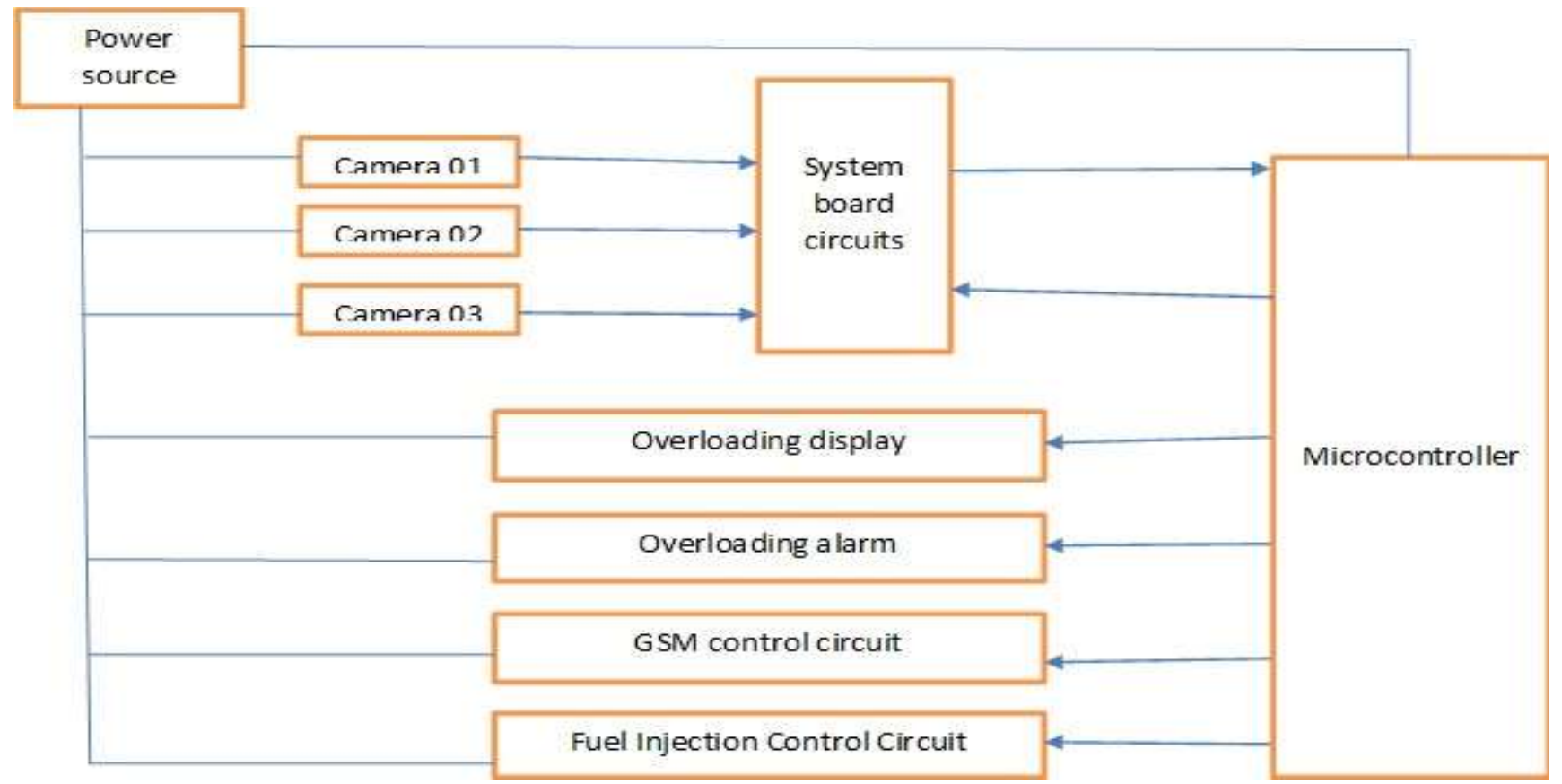

Figure 1: Block diagram for vehicles' overloading control system

\subsubsection{Use case}

Use case diagram usually used describes the main system functions from the standpoint of an external observer, created during the early stages of a project mostly during the analysis phase so as to demonstrate the different ways of user might use to interact with a system. The figure 2 below explain the use case diagram of the passenger's overloading control system.

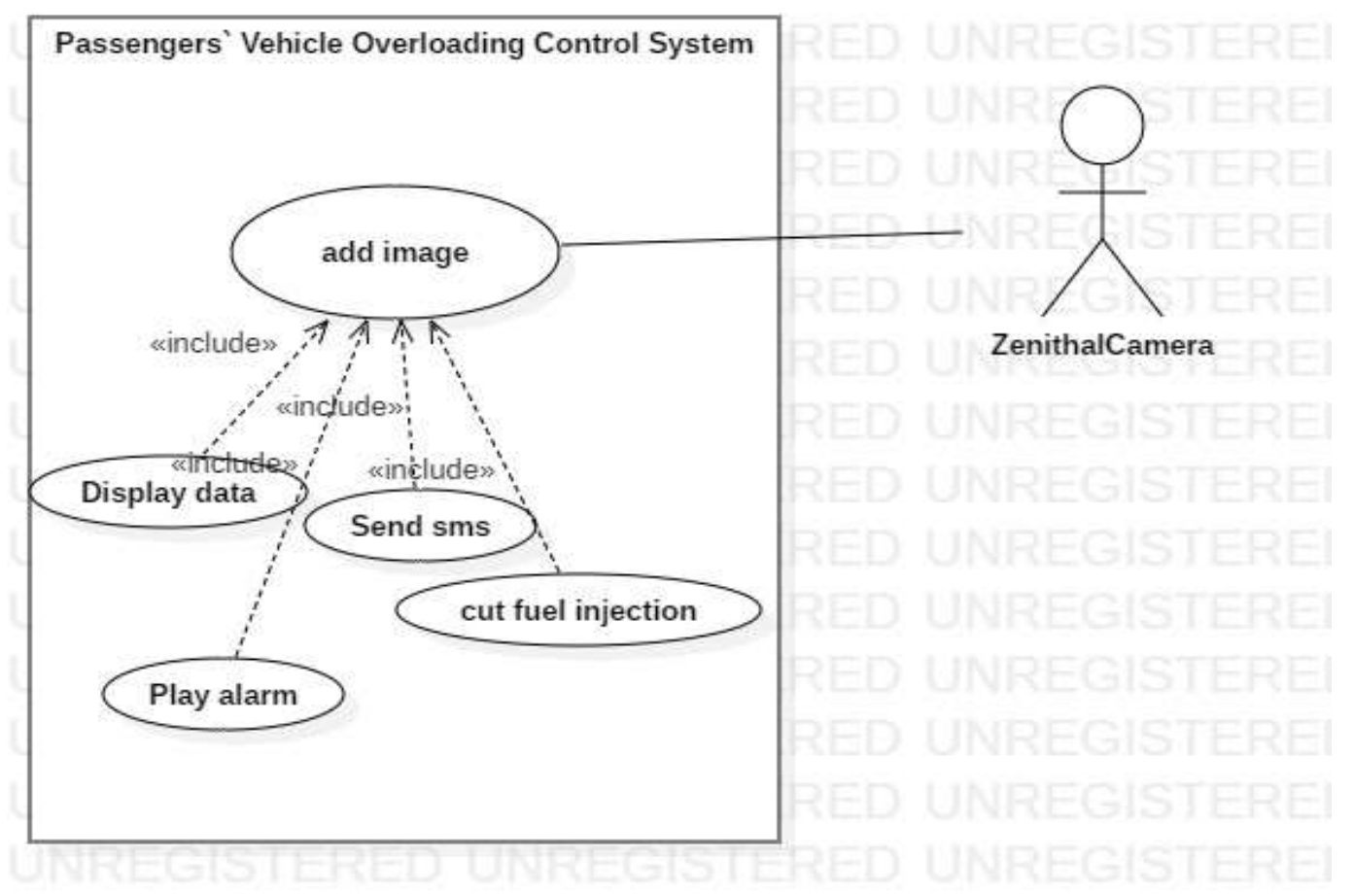

Figure 2: Use case diagram for the vehicles' overloading control system 


\subsubsection{Sequence diagram}

Is a system interaction model, it is also called the event diagram and is used to model the interactions between the actors_and the objects within a system. The figure 3 below enlighten the sequence diagram of the passenger's overloading control system

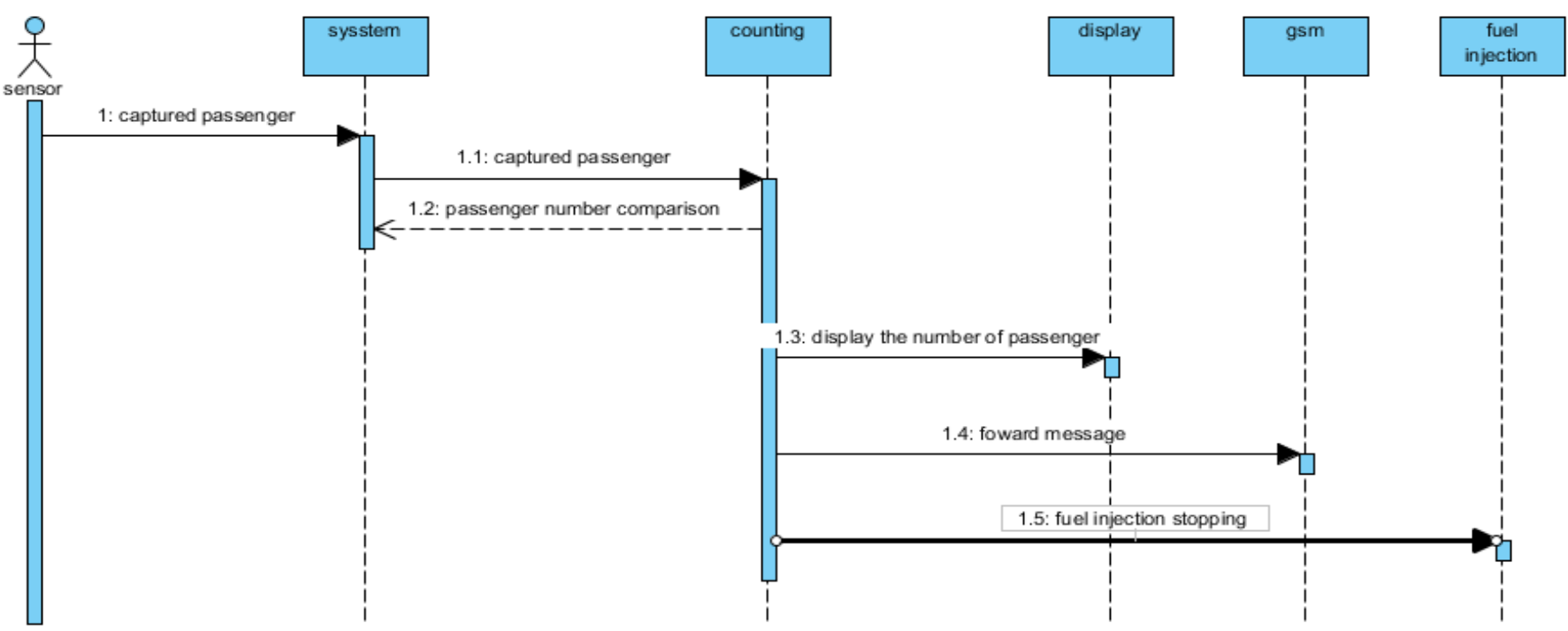

Figure 3: Sequence diagram for the vehicles' overloading control system

\subsection{The prototyping design}

The prototype is draft version of fabricated system on PCB board that shows general design concept, test the idea and show intention of investing money for product development process. Prototype is considered as a first or preliminary version of a device or vehicle from which other forms are developed. The prototype under consists camera that capture image of passenger and sending this image to the Arduino microcontroller that analyze the image and count the number of passenger for decision making and responding accordingly. ADCUM Camera, Diode 1N4007, Arduino UNO R3, GSM modem A6, SIM900 Liquid crystal display 16X4, DC motor, Relay 5v, Regulator 7805 and battery 4AH have been used to develop the system prototype. Figure 4 below is the diagram of the simulated circuit and figure 5 shows the developed prototype. 


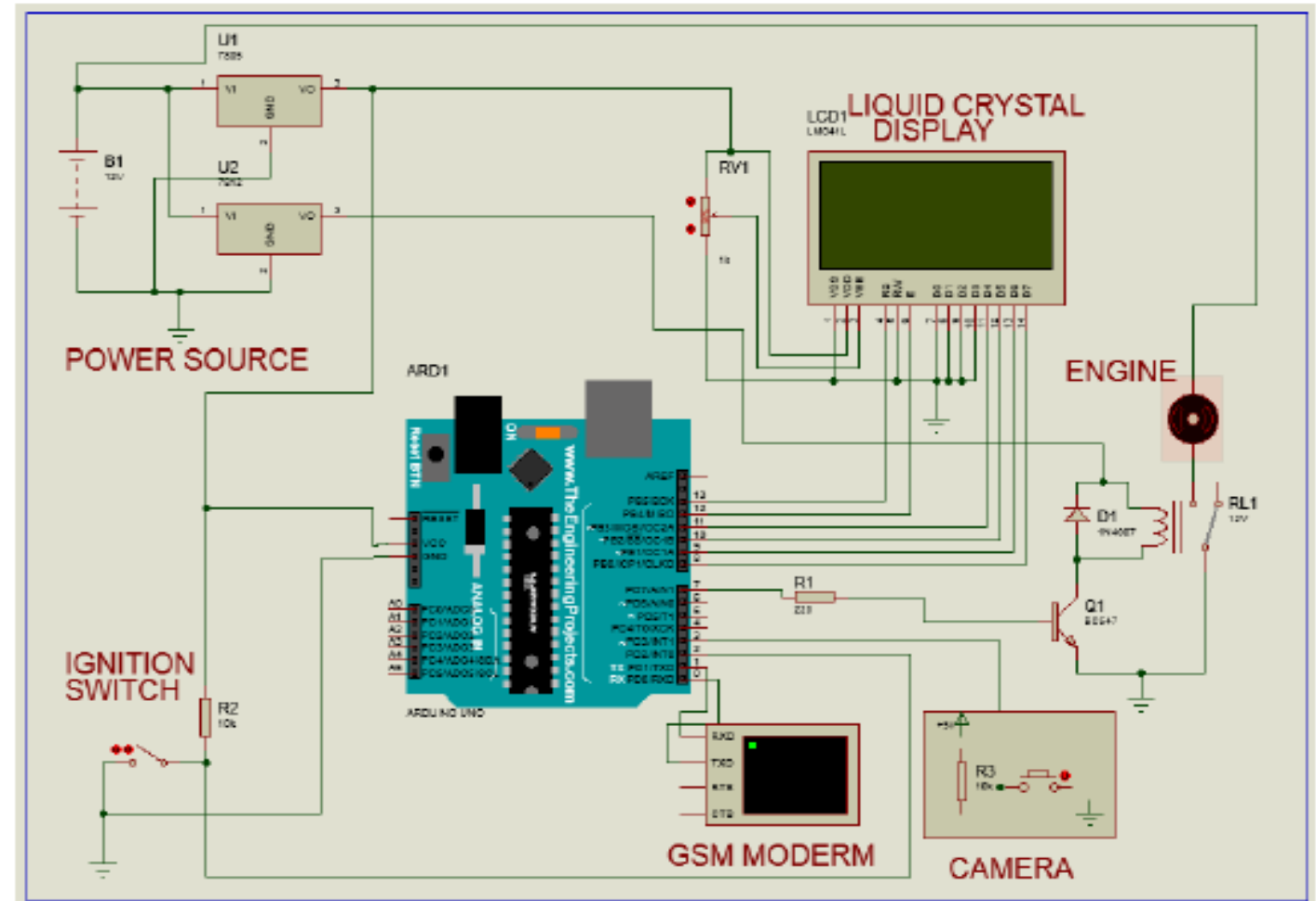

Figure 4: Circuit diagram for the passengers' overloading system

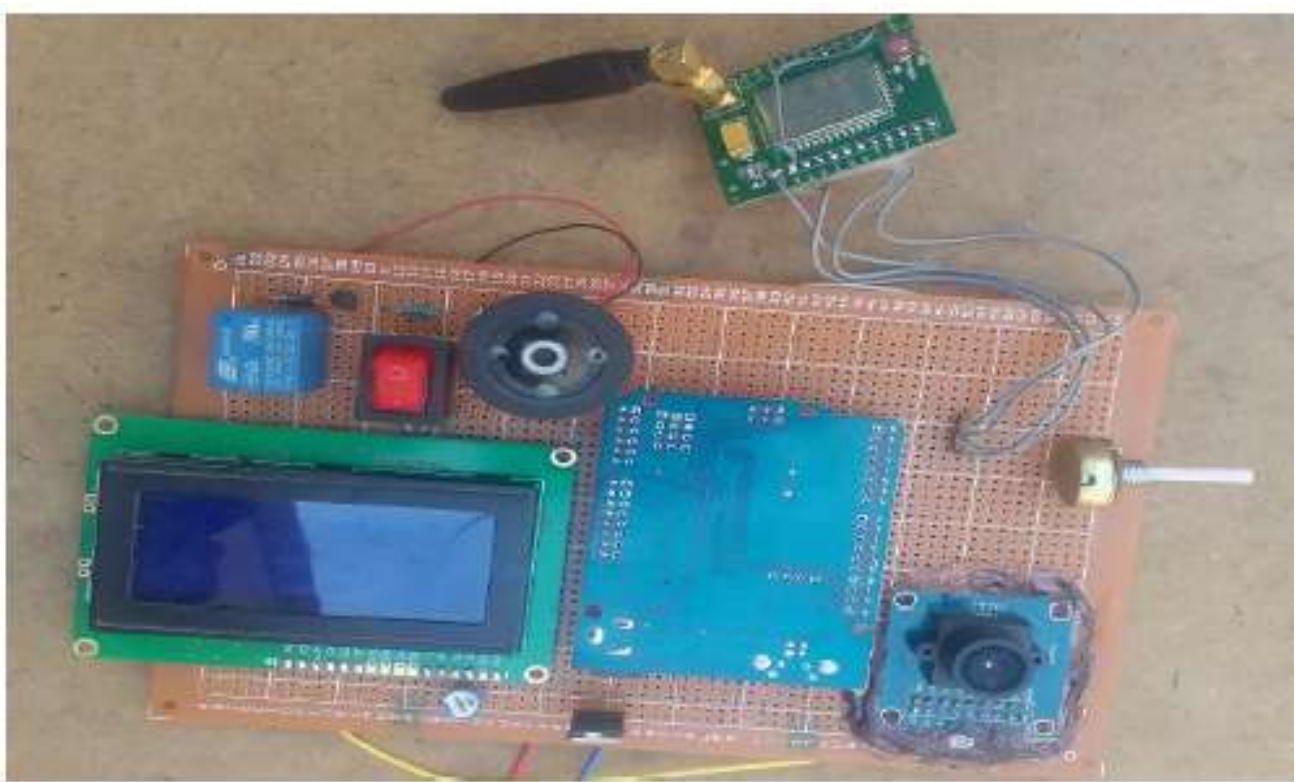

Figure 5: Prototype of the passengers' overloading system

\subsection{FINDINGS}

From the analysis of the data collected in the research, $65 \%$ of respondent indicated the need of developing sophisticated system responsible for automatically controlling public transport overloading in a real time. $92 \%$ confirmed that inspections are not done at night, $67 \%$ agreed on having means of bypassing checkpoints and $89 \%$ confirmed officers are not conducting inspections regularly. Early implementation of system to the Tanzania public transport industry is mandatory.

\subsection{CONCLUSIONS AND RECOMMENDATIONS}

\subsection{Conclusions}


Passengers`Vehicle Overloading Control System is the system used in bus passengers, the system minimize and reduce the incorrect information provided by manual system then control passenger overloading and replace manual system for automatic system. The data needed for design of the system are being successfully collected and analyzed and then used to build the prototype. When scaled and deployed in public transport the system may aid a proper management of the passengers overloading issue and reduce causalities in case accidents occur.

\subsection{Recommendations}

The intervention of the Tanzanian government on public transport in ensuring road safety has been promising, in recent years there has been the increase of traffic officers, vehicles' inspectors and establishment various agencies to control public transport related issues including the overloading. Accidents have been shrinking, however the number of causalities is still prominent. Drivers and other stakeholders in the industry have prolonged cheating on a number of public transport parameters including the issue of overloading.

The increase of inspection officers goes in line with the increase of expenses, the government is solving the problem at the expense of incurring more costs. We recommend the government to change its strategies and invest more on researches aiming at developing sophisticated systems like this which works in absence of human interventions and appeal for a valid data. More policies are to be developed insisting on operators to have systems of this kind.

\section{Acknowledgment}

The authors of this research paper acknowledge supports received from the Management of College of Engineering and Technology of Mbeya University of Science and Technology and other comments from reviewers.

\section{REFERENCES}

[1] H. Kilavo , S. Anael and D. Machuve, "CONTROL IN PUBLIC BUSESCASE STUDY: TANZANIA," International Journal Of Engineering And Computer Science ISSN:2319-7242, vol. 8, no. 2, pp. 2536-2540, 2013.

[2] E. Leonard, Traffic Safety and the Driver, London: Nostrand Reinhold Company, 1991.

[3] S. XU and Q. ZHAO, "Study on Vehicle-mounted Overloading Control System for Passenger Vehicles," ia Engineering 00 (2, vol. 15, pp. 1214 - 1218, 2011.

[4] I. Pinna, B. D. Chiara and F. Paolo , "Intelligent Transport," Intelligent Transport, 15 12 2010. [Online]. Available: https://www.intelligenttransport.com/transport-articles/3116/automatic-passenger-counting-systems-forpublic-transport/. [Accessed 2307 2019].

[5] Y. Tarek, K. Louahdi and M. Cyril, "Real-time passenger counting in buses using dense stereo vision," Journal of Electronic Imaging , vol. 19, no. 2, p. 031202, 2010.

[6] D. Beymer, "Person counting using stereo," in Workshop on HumanMotion, pp. 127-133, IEEE Computer Society, , Washington, DC, 2000.

[7] M. Rossi and A. Bozzoli, "Tracking and counting moving people," IEEE International Conference on Image Processing (ICIP), vol. 3, pp. 212-216, 1994.

[8] T.-H. C. Chao-Ho, C. Yin-Chan, C. Tsong-Yi and W. a-Jinn, "People Counting System for Getting In/Out of a Bus Based on Video Processing," in Eighth International Conference on Intelligent Systems Design and Applications, 
Kaohsiung, 2008.

[9] D. F. Denise and C. T. Beck, Nursing Research: Principles and Methods Seventh Edition, Philadelphia: jb lippincott, 2004.

[10] C. R. Kothari, Research Methodology: Methods and Techniques, india: New Age International, 2004.

[11] G. Marczyk, D. DeMatteo and . D. Festinger, Essentials of research design and methodology., Hoboken, NJ, US: John Wiley \& Sons Inc, 2005.

\section{List of Abbreviations}

MUST Mbeya University of Science and technology

LCD Liquid Crystal Display.

PVOCS Passengers' vehicle overloading control system

IC Integrated Circuit

JASP Jeffrey's Amazing Statistics Program

SUMATRA The Surface and Marine Transport Regulatory Authority

OOP Object-Oriented Programming

UML Unified Modelling Language

PCB Printed Circuit Board 